\title{
Evaluation of the effects of different culture media on the myogenic differentiation potential of adipose tissue- or bone marrow-derived human mesenchymal stem cells
}

\author{
JENS STERN-STRAETER ${ }^{1 *}$, GABRIEL ALEJANDRO BONATERRA ${ }^{2 *}$, STEPHANIE JURITZ $^{1}$, RICHARD BIRK ${ }^{1}$, \\ ULRICH REINHART GOESSLER ${ }^{1}$, KAREN BIEBACK ${ }^{3}$, PETER BUGERT ${ }^{3}$, JOHANNES SCHULTZ ${ }^{1}$, \\ KARL HÖRMANN $^{1}$, RALF KINSCHERF ${ }^{2 * *}$ and ANNE FABER ${ }^{1 * *}$ \\ ${ }^{1}$ Department of Otorhinolaryngology, Head and Neck Surgery, Medical Faculty Mannheim, University of Heidelberg, \\ 68167 Mannheim; ${ }^{2}$ Anatomy and Cell Biology, Department of Medical Cell Biology, 35032 Marburg; \\ ${ }^{3}$ Department of Transfusion Medicine and Immunology, Medical Faculty Mannheim, \\ University of Heidelberg, 68167 Mannheim, Germany
}

Received September 21, 2013; Accepted October 25, 2013

DOI: $10.3892 / \mathrm{ijmm} .2013 .1555$

\begin{abstract}
The creation of functional muscles/muscle tissue from human stem cells is a major goal of skeletal muscle tissue engineering. Mesenchymal stem cells (MSCs) from fat/adipose tissue (AT-MSCs), as well as bone marrow (BM-MSCs) have been shown to bear myogenic potential, which makes them candidate stem cells for skeletal muscle tissue engineering applications. The aim of this study was to analyse the myogenic differentiation potential of human AT-MSCs and BM-MSCs cultured in six different cell culture media containing different mixtures of growth factors. The following cell culture media were used in our experiments: mesenchymal stem cell growth medium (MSCGM) ${ }^{\mathrm{TM}}$ as growth medium, MSCGM + 5-azacytidine (5-Aza), skeletal muscle myoblast cell growth medium (SkGM)-2 BulletKit ${ }^{\mathrm{TM}}$, and 5, 30 and $50 \%$ conditioned cell culture media, i.e., supernatant of human satellite cell cultures after three days in cell culture mixed with MSCGM. Following the incubation of human AT-MSCs or BM-MSCs for $0,4,8,11,16$ or 21 days with each of the cell culture media, cell proliferation was measured using the alamarBlue ${ }^{\circledR}$ assay. Myogenic differentiation was evaluated by quantitative gene expression analyses, using quantitative RT-PCR (qRT-PCR) and immunocytochemical staining (ICC), using well-defined skeletal markers, such as desmin (DES),
\end{abstract}

Correspondence to: Dr Jens Stern-Straeter, Department of Otorhinolaryngology, Medical Faculty Mannheim, University of Heidelberg, Theodor-Kutzer-Ufer 1-3, 68167 Mannheim, Germany

E-mail: jens.stern-straeter@umm.de

*Contributed equally

${ }^{* *}$ Senior authorship

Key words: mesenchymal stem cell, skeletal muscle, tissue engineering, culture media myogenic factor 5 (MYF5), myosin, heavy chain 8, skeletal muscle, perinatal (MYH8), myosin, heavy chain 1, skeletal muscle, adult (MYH1) and skeletal muscle actin- $\alpha 1$ (ACTA1). The highest proliferation rates were observed in the AT-MSCs and BM-MSCs cultured with SkGM-2 BulletKit medium. The average proliferation rate was higher in the AT-MSCs than in the BM-MSCs, taking all six culture media into account. qRT-PCR revealed the expression levels of the myogenic markers, ACTA1, MYH1 and MYH8, in the AT-MSC cell cultures, but not in the BM-MSC cultures. The muscle-specific intermediate filament, DES, was only detected (by ICC) in the AT-MSCs, but not in the BM-MSCs. The strongest DES expression was observed using the $30 \%$ conditioned cell culture medium. The detection of myogenic markers using different cell culture media as stimuli was only achieved in the AT-MSCs, but not in the BM-MSCs. The strongest myogenic differentiation, in terms of the markers examined, was induced by the $30 \%$ conditioned cell culture medium.

\section{Introduction}

The formation of functional skeletal muscles/muscle tissue utilising the differentiation potential of stem cells is the intention of skeletal muscle tissue engineering. Due to the myogenic differentiation potential of human mesenchymal stem cells (MSCs), these cells are new candidate stem cells for tissue engineering applications, which bear certain advantages over previously utilised stem cells, such as satellite cells (1). MSCs can be extracted from various sources, such as bone marrow (1), adipose tissue (2), umbilical cord blood (3) or the placenta (4), and therefore multiple harvesting methods are possible. MSCs derived from fat/adipose tissue (AT-MSCs) or bone marrow (BM-MSCs) are characterised, in contrast to other stem cells, by their high potential for replication without losing their differentiation ability (5). Thus, greater amounts of neo-tissue could be generated by smaller donor cell populations. Moreover, MSCs can be transplanted autologously and can enhance tissue regeneration due to immunomodula- 
tion (6,7). These attributes make MSCs ideal stem cells for tissue engineering.

The myogenic differentiation of MSCs can be initiated with different growth factors, such as basic fibroblast growth factor (bFGF), platelet-derived grow th factor (PDGF), the amino acid, L-glutamine, and 5-azacytidine (5-Aza), which can all be added to the growth medium $(5,8)$. The exact molecular mechanisms associated with the intracellular signalling cascades which control the differentiation process remain unclear (9). The influence of growth factors on cell differentiation and proliferation varies according to the type, strain and origin of the MSCs (10). Another important factor is the degree of activation of the Rho GTPase, which can control the differentiation in myogenic or adipogenic cell lines (11). Therefore, in this study, we evaluated the proliferation and myogenic differentiation potential of human AT-MSCs and BM-MSCs cultured in six different culture media with different growth factor compositions, in order to detect possible differences between the two stem cell types. In this context, we used the following cell culture media: mesenchymal stem cell growth medium $(\mathrm{MSCGM})^{\mathrm{TM}}$ by Lonza ${ }^{\circledR}$ as growth medium, MSCGM + 5-Aza, skeletal muscle myoblast cell growth medium (SkGM)-2 BulletKit ${ }^{\mathrm{TM}}$, and 5, 30 and $50 \%$ conditioned cell culture media, i.e., supernatant of human satellite cell cultures after three days in cell culture mixed with MSCGM.

During the transformation from mononucleated myoblasts into multinucleated myofibers, multiple muscle-specific genes are switched on and can therefore act as specific markers of the different stages of myogenesis. As an early marker of differentiation, we investigated the transcription factor, myogenic factor 5 (MYF5), as it acts as promoter of multiple muscle-specific genes, controls the fusion of multinucleated myofibers (12), and is upregulated during the early stages of myogenesis $(13,14)$. Genes of the contractile apparatus served as late markers of differentiation. Myosin heavy chain (MYH) is part of the hexameric myosin complex, which consists of four light chains and two heavy chains and constitutes up to $50 \%$ of the total protein content of adult skeletal muscle tissue $(14,15)$. MYH participates in the contraction processes and exists in multiple isoforms [myosin, heavy chain 8, skeletal muscle, perinatal (MYH8) and myosin, heavy chain 1, skeletal muscle, adult (MYH1)], which can serve as markers of differentiation $(14,16)$. As a marker of the terminal stages of myogenesis, actin- $\alpha 1$ (ACTA1) is an important protein of the contractile apparatus in skeletal muscle tissue (14). In order to evaluate the effects of culture with six different cell culture media on the proliferation of human AT-MSCs and BM-MSCs, we performed alamarBlue ${ }^{\circledR}$ proliferation assays from day 0 to day 21 . In order to analyse the effects of the different cell culture media on the myogenic differentiation of AT-MSCs and BM-MSCs, quantitative reverse transcription polymerase chain reaction (qRT-PCR) was performed to determine the gene expression levels of MYF5, MYH8, MYH1 and ACTA1. As a prerequisite, suitable reference genes for qRT-PCR experiments needed to be identified. Therefore, we evaluated the expression stability of $\beta$-actin (ACTB), $\beta$-2-microglobulin (B2M), glyceraldehyde-3-phosphate dehydrogenase (GAPDH), peptidylprolyl isomerase A (PPIA), TATA-box binding protein (TBP) and ribosomal protein, large, P0 (RPLPO) in human MSCs using two different software programs, geNorm and NormFinder. Additionally, immunocytochemistry (ICC) was performed to determine the expression of desmin (DES), MYF5 and ACTA1, to verify myogenic differentiation.

Thus, our study presents novel data on the myogenic differentiation potential of human MSCs derived from bone marrow and adipose tissue, utilising six different cell culture media with different growth factor compositions.

\section{Materials and methods}

Collection and isolation of human MSCs derived from bone marrow. Human MSCs were isolated from adult bone marrow as previously described by Goessler et al (17). Bone marrow was collected from the femoral shaft of patients undergoing total hip replacement at the Orthopaedic Department of the University Hospital Mannheim, Mannheim, Germany. The study protocol was approved by the Ethics Committee of the Mannheim Medical Faculty of the University of Heidelberg, Heidelberg, Germany. Bone marrow aspirates were diluted 1:5 with PBS/2 mM EDTA (Nexell, Baxter, Unterschleissheim, Germany and Merck, Darmstadt, Germany) and carefully loaded into Ficoll-Hypaque solution (Amersham, Freiburg, Germany). Mononuclear cells (MNCs) were isolated after density gradient centrifugation at $435 \mathrm{x}$ g for $30 \mathrm{~min}$ at room temperature. The MNCs were then removed from the interphase, washed two to three times with PBS/EDTA and set in culture at a density of $1 \times 10^{6}$ cells $/ \mathrm{cm}^{2}$. Cell counts were performed using an automated cell analyser (Cell-Dyn 3200, Abbott, Wiesbaden, Germany). Cells were grown in MSCGM BulletKit, Cambrex, St. Katharinen, Germany). Non-adherent cells were removed by the complete exchange of culture medium every three to four days. The flasks were screened continuously to access developing colonies of adherent cells. Fibroblastoid cells were recovered between days seven and ten after initial plating using $0.04 \%$ trypsin/0.03\% EDTA (PromoCell, Heidelberg, Germany) and termed bone marrowderived fibroblastoid adherent cells (BM-FACs). Recovered cells were plated at a density of $4,000-5,000$ cells $/ \mathrm{cm}^{2}$ as passage 1 cells, as previously described (17).

\section{Collection and isolation of human MSCs derived from adipose} tissue. Lipoaspirates were obtained from patients undergoing liposuction procedures in accordance with the standard of ethics of the local ethics committee. The raw lipoaspirates $(50-100 \mathrm{ml})$ were processed as described previously by Goessler et al (17). To isolate the stromal vascular fraction (SVF), lipoaspirates were washed with PBS and digested with an equal volume of $0.075 \%$ collagenase type I (Sigma-Aldrich, St. Louis, MO, USA) for $30-60 \mathrm{~min}$ at $37^{\circ} \mathrm{C}$. The collagenase activity was blocked with DMEM-lg containing $10 \%$ FCS and the lipoaspirates were centrifuged at $1,200 \mathrm{x}$ for $10 \mathrm{~min}$ to harvest a SVF pellet. The pellet was re-suspended in MSCGM and filtered through a $100 \mu \mathrm{m}$ nylon cell strainer (BD Falcon, Franklin Lakes, NJ, USA). The filtered cells were centrifuged at $1,200 \mathrm{x}$ g for $10 \mathrm{~min}$. The SVF cell suspension was plated at a density of $1 \times 10^{6} / \mathrm{cm}^{2}$ into T75 or T175 culture flasks. Non-adherent cells were removed after $12-18$ h by PBS washings. The resulting fibroblastoid adherent cells were termed adipose tissue-derived fibroblastoid adherent cells (AT-FACs). 
AT-FACs were harvested at approximately $70 \%$ confluence using trypsin. On the second pass, cells were replated at a mean density of $1.8 \pm 3.1 \times 10^{3} / \mathrm{cm}^{2}$.

Cultivation of human MSCs derived from bone marrow and adipose tissue in different culture media

MSCGM by Lonza ${ }^{\circledast}$. MSCGM (Lonza, Verviers, Belgium) is a growth medium, which was designed for the cultivation of MSCs. This cell culture medium is purchased as a kit and contains the amino acid, L-glutamine, and mesenchymal stem cell growth supplement (MSCGS). The precise composition is only known to the manufacturer.

$M S C G M+5-A z a .5$-Aza (Sigma-Aldrich, Irvine, UK) was added at a concentration of $10 \mu \mathrm{mol} / 1$. Additionally, $10 \mu \mathrm{g} / \mathrm{l}$ human, recombinant fibroblast growth factor (rhFGF) (Lonza) was appended.

SkGM-2 BulletKit. The SkGM-2 BulletKit (SKM, Lonza) is a commercial basal medium for MSCs, which includes pro-myogenic factors that induce myogenic differentiation. The constituents of the medium are: Skm-medium, FBS, L-glutamine, dexamethasone, rhFGF and GA-1000 (gentamycin/amphotericin 1:1,000).

Conditioned cell culture medium. The conditioned cell culture medium contains the supernatant of human satellite cells under differentiation conditions using serum cessation, which were grown in Ham's F10 culture medium. The supernatant was removed after three days, filtered for sterility and mixed to 5,30 and $50 \%$ concentrations with growth medium.

alamarBlue proliferation assay. AT-MSCs and BM-MSCs were cultured in $0.2 \%$ gelatine-coated 96 -well plates at a density of 5,000 cells/well in MSCGM, MSCGM + 5-Aza, SkGM-2 BulletKit, 5, 30 and 50\% conditioned cell culture medium. Proliferation was assessed by measurement of the fluorescence at wavelength of $540 \mathrm{~nm}$ and emission was monitored at $590 \mathrm{~nm}$ on days $0,4,8,11,16$ and 21 .

RNA isolation. The RNeasy mini kit (Qiagen, Hilden, Germany) was used according to the manufacturer's instructions to isolate total RNA. RNA purity and concentration were determined by measurements at A260 and A280 nm (A260/A280, 1.7-2.0) using a NanoDrop 8000 Spectrophotometer (Thermo Scientific, Schwerte, Germany). RNA integrity was determined using a 2100 Bioanalyzer (Agilent Technologies, Waldbronn, Germany).

cDNA synthesis and qRT-PCR. cDNA synthesis and qRT-PCR were performed as previously described (18). An aliquot of $0.5 \mu \mathrm{g}$ total RNA was treated with 1 unit DNase (Fermentas, St. Leon-Rot, Germany) for $30 \mathrm{~min}$ at $37^{\circ} \mathrm{C}$. Reverse transcription of RNA $(0.5 \mu \mathrm{g})$ was performed using oligo $(\mathrm{dT})_{12-18}$ primer and 200 units of SuperScript II(Invitrogen, Karlsruhe, Germany) and 24 units of RiboLock ${ }^{\text {TM }}$ RNase inhibitor (Fermentas) for $1 \mathrm{~h}$ at $42^{\circ} \mathrm{C}$. The cDNA was used for qRT-PCR analysis. All cDNA probes were analysed for MYF5, NM_002479; ACTA1, NM_001100; MYH1, NM_005963; and MYH8, NM_002472; as well as the reference genes: ACTB, NM_001101; B2M, NM_004048; GAPDH, NM_002046; PPIA (cyclophilin A), NM_203430; RPLPO, NM_001002; and TBP, NM_003194. The QuantiTect/PrimerAssays were purchased from Qiagen. cDNA was amplified with Brilliant ${ }^{\circledR}$ II SYBR ${ }^{\circledR}$-Green
qRT-PCR Master Mix (Stratagene-Agilent Technologies, Waldbronn, Germany). The thermal profile consisted of 1 cycle at $50^{\circ} \mathrm{C}(2 \mathrm{~min})$ followed by 1 cycle at $95^{\circ} \mathrm{C}(2 \mathrm{~min})$, 45 cycles at $95^{\circ} \mathrm{C}(15 \mathrm{sec})$ and $60^{\circ} \mathrm{C}(1 \mathrm{~min})$. Amplification was performed using the Mx3005P ${ }^{\mathrm{TM}}$ QPCR System (Stratagene). For relative quantification, a serial dilution of the cDNA was used to perform a standard curve in every individual run. The data were analysed using the relative standard curve method. For each unknown sample, the relative amount was calculated using linear regression analysis from their respective standard curves. Data were analysed using Mx3005P analysis software (Stratagene-Agilent Technologies). The efficiencies of all genes of interest, including the reference genes were calculated for each individual run.

Analysis of expression stability. Expression stability was calculated as previously described (18). The qRT-PCR data were analysed using the Mx3005P QPCR System (Stratagene). For stability comparisons of candidate reference genes within sample groups, the software geNorm (19), version 3.4 (Visual Basic application tool for Microsoft Excel), was used according to the developer's recommendations.

GeNorm uses a gene-stability measurement, $\mathrm{M}$, which is defined as the average pair wise variation between a particular gene and all other control genes. It calculates the optimal number of genes required for the normalisation of a target gene and combines them into a normalisation factor (NF) $(19,20)$. The expression of the genes of interest was normalised to the NF.

Immunocytochemistry (ICC). Immunocytochemical characterisation was carried out as previously described (18). The cells were grown on chamber culture slides (BD Falcon). To indicate the differentiation of the MSCs, the cells were incubated with antibodies directed against: DES (Dako, Hamburg, Germany), MYF5 (Santa Cruz, Biotechnology, Heidelberg, Germany) and ACTA1 (Zymed Laboratories, Invitrogen, Karlsruhe, Germany). The antibodies were used according to the manufacturer's instructions, followed by a corresponding biotinylated secondary antibody and streptavidin, horseradish peroxidase (HRP) conjugate. The peroxidase reaction was performed using aminoethylcarbazole (Dako) as a chromogen. Endogenous peroxidase was blocked with $0.3 \%$ hydrogen peroxide $(30 \mathrm{~min})$. Sections were washed with PBS and incubated with normal sheep serum in PBS (3 min, room temperature) to block nonspecific antibody reaction. Nuclei were counterstained with Harris haematoxylin. Light microscopic investigations were performed using a Zeiss Axiophot microscope (Carl Zeiss, Jena, Germany).

\section{Results}

Proliferation of human AT-MSCs cultured in different cell culture media. Cell proliferation was measured using the alamarBlue proliferation assay from day 0 to day 21 (Fig. 1). No cytotoxic effects were observed during cultivation in any of the cell culture media (data not shown). AT-MSCs grown in the SkGM-2 BulletKit showed the highest proliferation rate compared with those grown in the other cell culture media analysed (Fig. 1). The fluorescence units (FU) increased by 


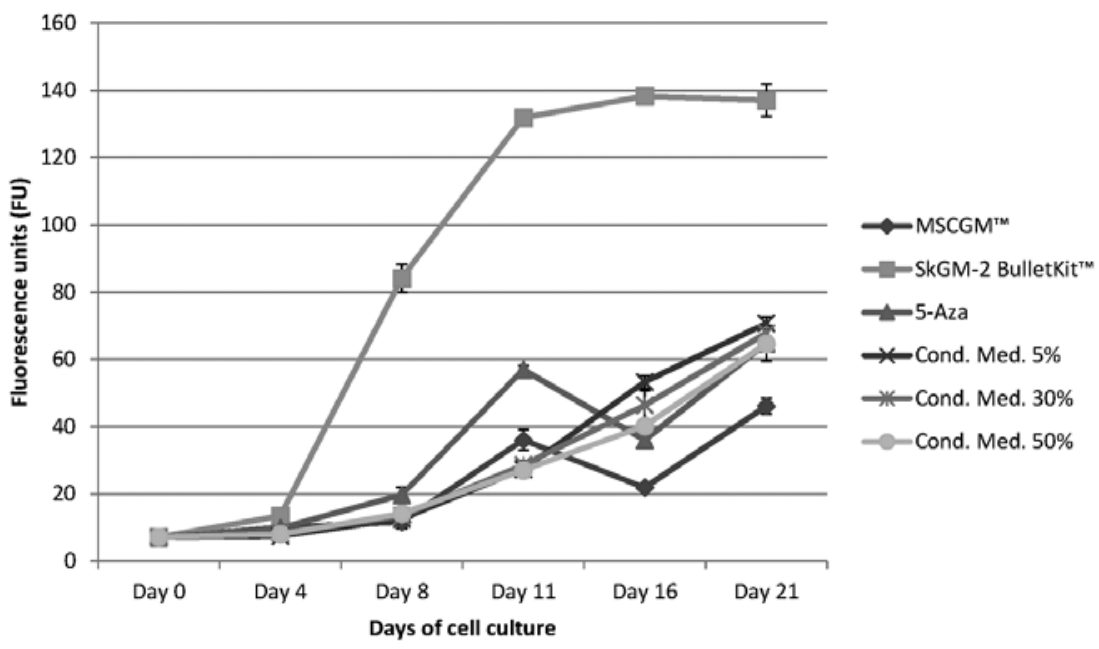

Figure 1. alamarBlue proliferation assay of human mesenchymal stem cells (MSCs) derived from fat/adipose tissue (AT-MSCs), which were nourished in different cell culture media. Cond. Med., conditioned medium.

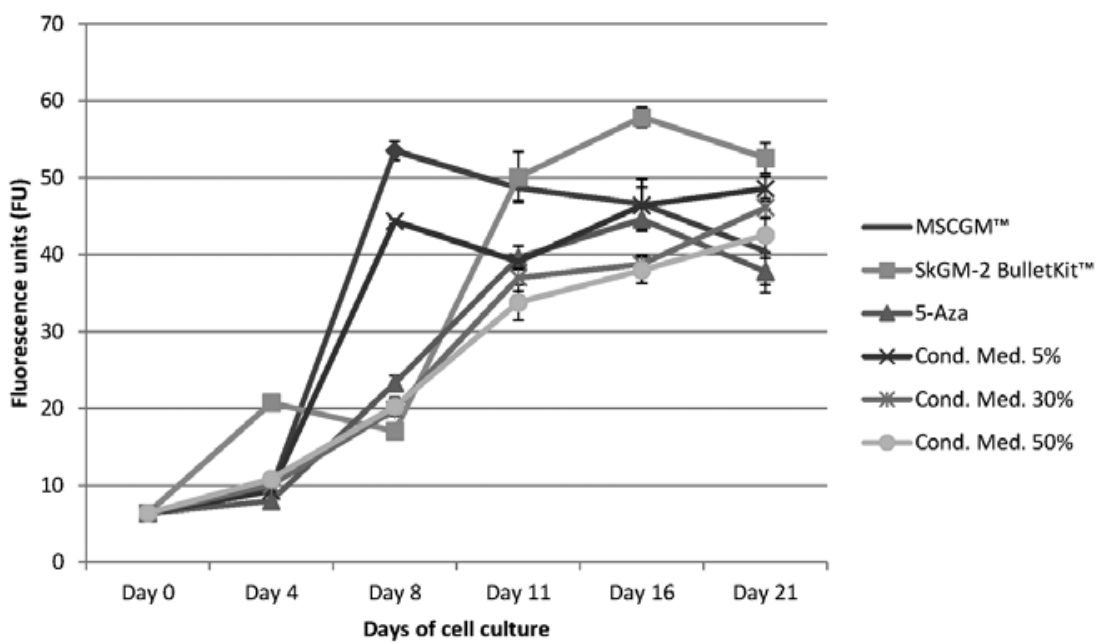

Figure 2. alamarBlue proliferation assay of human mesenchymal stem cells (MSCs) derived from bone marrow (BM-MSCs), which were nourished in different cell culture media. Cond. Med., conditioned medium.

approximately 20 -fold from $7.27 \pm 0.29$ on day 0 continuously to the maximum of $138.2 \pm 1.66$ on day 16 . After this time point, the proliferation almost remained on this high level on day 21 and was approximately 2-2.5-fold higher than that of the AT-MSCs cultured in one of the other five media (Fig. 1).

The conditioned cell culture media showed, independent of their concentration (5, 30 and 50\%), almost identical rates of cell proliferation. The FU level began at $7.27 \pm 0.29$ on day 0 and continuously increased to a maximum level on day 21 (Fig. 1). After 21 days of cell culture, the FU values for the conditioned cell culture media were similar: 70.71 $\pm 1.88(5 \%), 67.78 \pm 2.07(30 \%)$ and $64.61 \pm 2.26(50 \%)$, and thus, were increased by $9-10$-fold in comparison to day 0 (Fig. 1). The stimulation of AT-MSCs with MSCGM + 5-Aza showed an 8-fold increase in the FU value from day 0 to day 11 , followed by a $35 \%$ decrease on day 16 and a renewed increase to a maximum level on day 21 (Fig. 1). After 21 days of cell culture, the FU values were $46.09 \pm 2.39$ for the AT-MSCs incubated with MSCGM and 64.81 \pm 5.2 following stimulation with MSCGM + 5-Aza, and were thus increased by 9-fold in comparison to day 0 (Fig. 1).
Proliferation of human BM-MSCs cultured in different cell culture media. BM-MSCs incubated with the SkGM-2 BulletKit medium revealed the highest proliferation rates compared with those grown in the other cell culture media (Fig. 2). The initial FU value on day 0 was $6.36 \pm 0.05$ and increased by approximately 9-fold, reaching the maximum value on day 16 (FU, 57.88 \pm 1.26 ) with the exception of a slight decrease on day 8 (Fig. 2).

Following culture with MSCGM, the BM-MSCs showed a steep 8.4-fold increase in proliferation, represented by an FU value of $53.51 \pm 1.23$ until day 8 , followed by a steady decline of cell turn over on days 11 and 16 until day 21 (-25\%), represented by a final FU value of $40.47 \pm 5.43$ (Fig. 2).

During the cultivation of BM-MSCs in the different conditioned $(5,30$ and $50 \%)$ cell culture media, different cell proliferation rates were measured, depending on the concentration of the medium. The FU value of cells grown in $5 \%$ conditioned cell culture medium increased by approximately 7 -fold until day 8 to an FU value of $44.33 \pm 0.33$, followed by a marginal decline $(-12 \%)$ of the FU value to $39.07 \pm 0.79$ on day 11 and an increase $(24 \%)$ in proliferation until day 21 with an FU 


\section{Averange expression stability values of reference genes in} MSCs

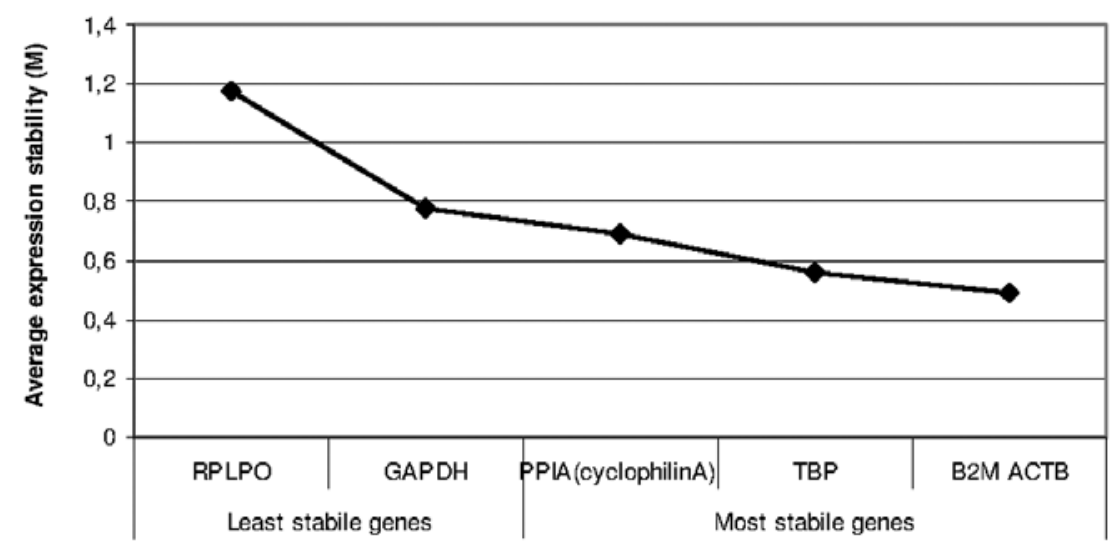

Figure 3. Average expression stability values of the reference candidate genes examined. The gene expression stability of six candidate reference genes was calculated using geNorm software to select the most stable gene for normalisation in human mesenchymal stem cells (MSCs). The average expression stability, M, was calculated by the software. The $\mathrm{x}$-axis from left to right indicates the ranking of the genes according to their M-value.

value value of $48.57 \pm 1.64$ (Fig. 2). In the BM-MSC cultures nourished with 30 or $50 \%$ conditioned cell culture medium, a similar time-dependent increase in proliferation was detected (Fig. 2). The proliferation rate increased steadily by approximately 7-fold from day 0 to day 21 . The FU value was $10.03 \pm 0.15$ (30\% conditioned cell culture medium) and $10.80 \pm 0.10$ (50\% conditioned medium) on day 4 and $19.85 \pm 0.25$ (30\% conditioned medium) and $20.26 \pm 1.19$ (50\% conditioned medium) on day 8 . The FU value increased further to $46.11 \pm 1.23$ on day 21 in the cells cultured in $30 \%$ conditioned medium and $42.52 \pm 2.16$ in those cultured in $50 \%$ conditioned medium (Fig. 2).

Identification of valid reference genes for qRT-PCR experiments. To identify the most stable reference gene for the normalisation of gene expression in qRT-PCR measurements, the following genes were analysed during the cultivation of the human AT-MSCs/BM-MSCs using the different culture media: ACTB, B2M, GAPDH, PPIA, TBP and RPLPO. The expression stability was calculated using the geNorm and NormFinder software.

geNorm. The geNorm program calculated that B2M and ACTB are the most robust reference genes. The results of the expression stability measurements are illustrated in Fig. 3.

NormFinder. The NormFinder software identified B2M and ACTB as the most stable reference genes (Table I).

Gene expression analyses using $q R T-P C R$. In the human AT-MSCs, only the expression of the myogenic markers, ACTA1, MYH8 and MYH1 could be detected using qRT-PCR. However, the expression of myogenic markers examined could not be detected in the human BM-MSCs.

ACTA1. The incubation of AT-MSCs with MSCGM + 5-Aza led on day 4 and on day 8 to an 1.2- and 2-fold increase in ACTA1 gene expression, respectively, compared with the cells cultured with MSCGM alone, followed by a decrease of $50 \%$ on day 11 and another 1.6-fold increase on day 16 (Fig. 4). On day 21, ACTA1 expression was almost similar to that found in the cells incubated with MSCGM alone (Fig. 4). The cells cultured with SkGM-2 medium showed a 2.3-fold increase
Table I. Stability measurements of the candidate reference genes in human MSCs.

\begin{tabular}{lcc}
\hline Gene name & Stability value & Standard error \\
\hline RPLPO & 1.996 & 0.260 \\
TBP & 0.355 & 0.100 \\
GAPDH & 0.496 & 0.104 \\
B2M & 0.307 & 0.101 \\
ACTB & 0.288 & 0.102 \\
PPIA & 0.467 & 0.103
\end{tabular}

Measurements were calculated by the NormFinder software. ACTB was identified as the most stable gene, where B2M ranked second. MSCs, mesenchymal stem cells; RPLPO, large ribosomal protein P0; TBP, TATA-box binding protein; GAPDH, glyceraldehyde-3-phosphate dehydrogenase; B2M, $\beta$-2-microglobulin; ACTB, $\beta$-actin; PPIA, peptidylprolyl isomerase A.

in ACTA1 expression on day 8 and a 1.6-fold increase on day 16 compared with the cells cultured with MSCGM alone. Incubation with $30 \%$ conditioned medium led to the highest relative expression compared to incubation with MSCGM, with an 3.8- and 1.9-fold increase on day 8 and on day 16, respectively. In the AT-MSCs nourished with $50 \%$ conditioned medium, the maximum ACTA1 expression was measured on day 4 with a 1.4-fold increase compared with the MSCGMcultured cells (Fig. 4).

MYH8. Gene expression analysis detected MYH8 in each of the six evaluated groups of human AT-MSCs cultured with the different cell culture media. Measured values showed very heterogeneous results in each culture medium. Trends were not observed. The highest gene expression was measured on day 21 with a concentration-dependent effect of the conditioned medium with a maximum 200 -fold increase using the $50 \%$ conditioned cell culture medium (Fig. 5). The highest MYH 8 gene expression for the cells cultured 


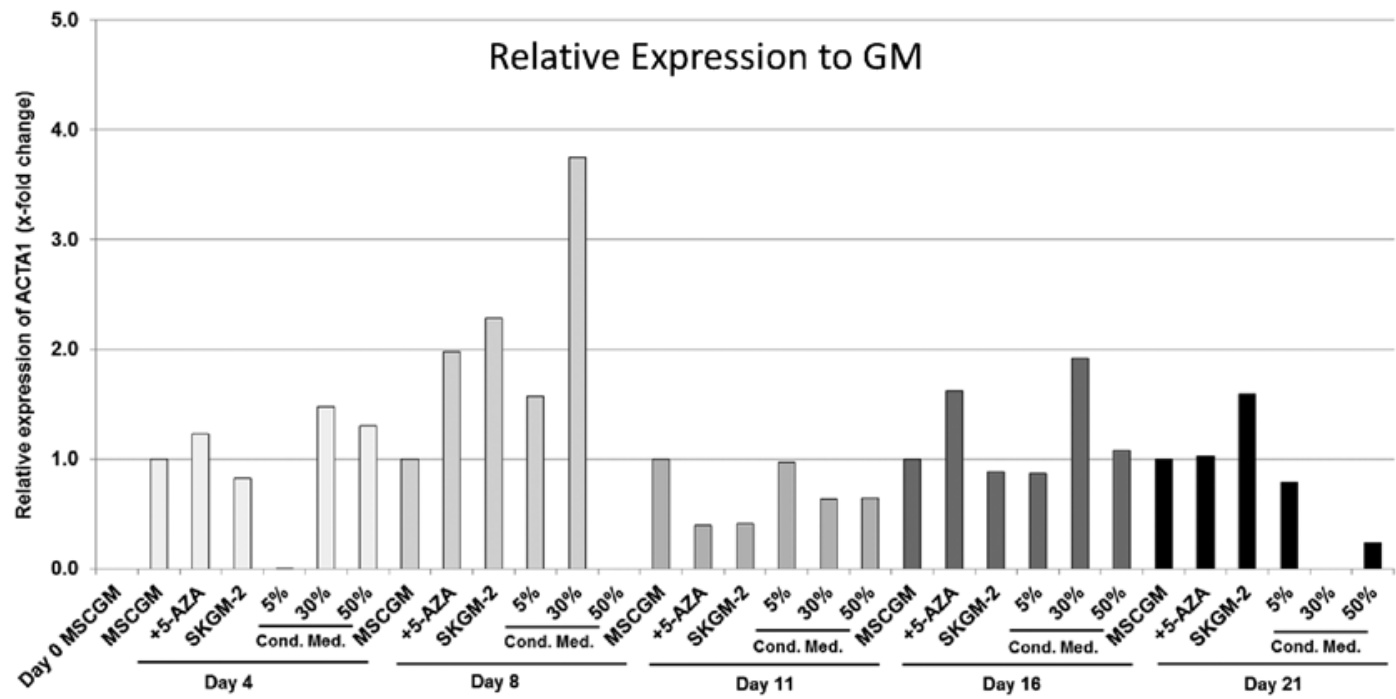

Figure 4. Expression analyses (qRT-PCR) of the skeletal muscle actin- $\alpha 1$ (ACTA1) gene in human mesenchymal stem cells (MSCs) derived from fat/adipose tissue (AT-MSCs). Gene expression is presented relative to the expression in human AT-MSCs incubated in growth medium (MSCGM). The genes were normalised using the normalisation factor (NF) calculated by geNorm software. MSCGM, MSCGM growth medium (GM); 5-Aza, MSCGM + 5-azacytidine (5-Aza); SkGM-2, SkGM-2 BulletKit; 5, 30, 50\% conditioned cell culture media, supernatant of human satellite cell cultures after three days in cell culture mixed with MSCGM. Cond. Med., conditioned medium.

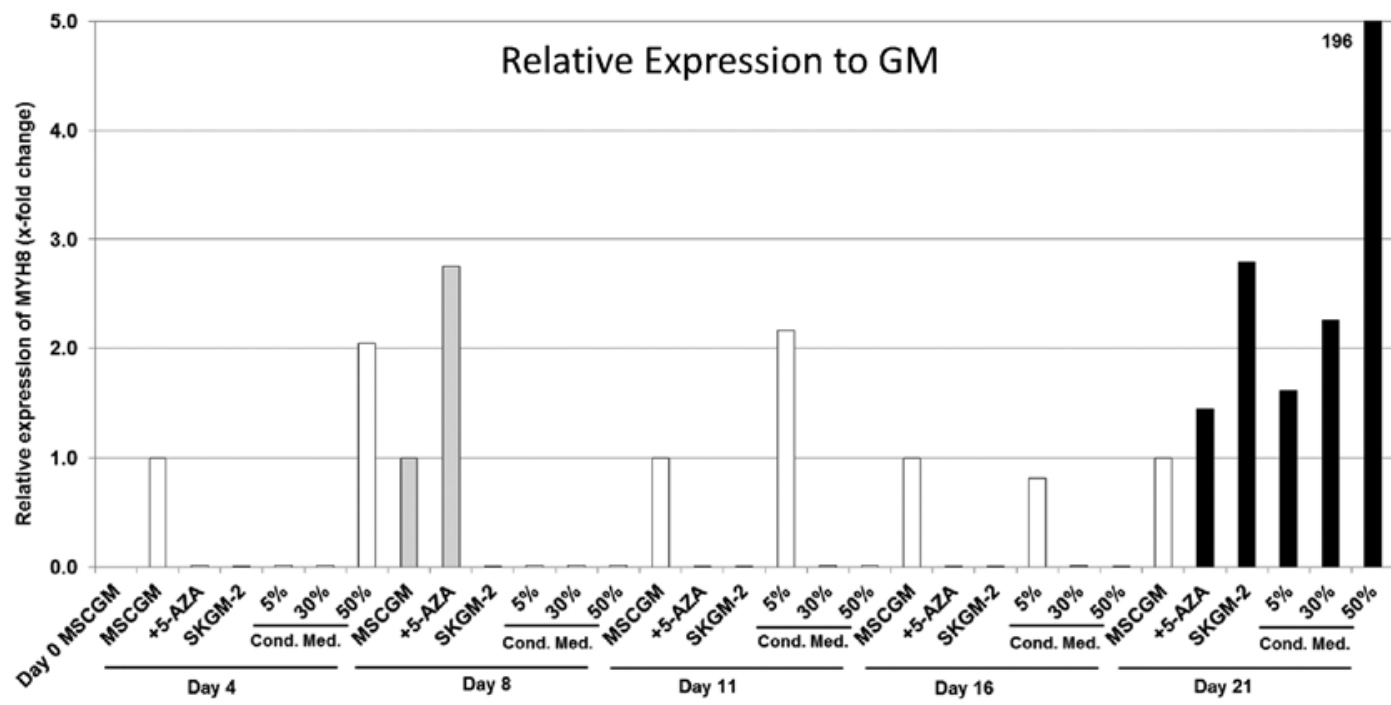

Figure 5. Expression analyses (qRT-PCR) of the myosin, heavy chain 8, skeletal muscle, perinatal (MYH8) gene in human mesenchymal stem cells (MSCs) derived from fat/adipose tissue (AT-MSCs). Gene expression is presented relative to the expression in human AT-MSCs incubated in MSCGM. The genes were normalised using normalisation factor (NF) calculated by geNorm software. MSCGM, MSCGM growth medium (GM); 5-Aza, MSCGM + 5-azacytidine (5-Aza); SkGM-2, SkGM-2 BulletKit; 5, 30, 50\% conditioned cell culture media, supernatant of human satellite cell cultures after three days in cell culture mixed with MSCGM. Cond. Med., conditioned medium.

in MSCGM + 5-Aza was detected on day 8 with a 2.7 -fold increase compared with the MSCGM-nourished cells (Fig. 5). The maximum values for cells cultured in SkGM-2 was measured on day 21 (2.7-fold higher compared with MSCGMcultured cells).

MYH1. The expression of MYH1 in the human AT-MSCs also showed very diverse results with the different culture media. The highest levels of expression of MYH1 were detected in the AT-MSCs nourished with SkGM-2 BulletKit (57-fold increase), as well as in those cultured with 5,30 and $50 \%$ conditioned medium (7.7-, 115- and 112-fold increase, respectively) on day 16 (Fig. 6).
Immunocytochemistry. To evaluate the myogenic differentiation of the human MSCs, immunocytochemical staining with antibodies directed against DES, MYF5 or ACTA1 was performed.

First of all, there was no ACTA1 immunoreactivity found in the human AT-MSCs incubated with each of the six media examined. However, the human AT-MSCs grown in conditioned cell culture media showed positive staining for the myogenic markers, DES and MYF5, after day 4 in cell culture (Fig. 7). The percentage of DES- and MYF5-positive AT-MSCs (Table II) increased in a time-dependent manner following culture in the $5 \%$ conditioned cell culture medium. 
Table II. Immunocytochemical evaluation of the muscle-specific differentiation markers, DES and MYF5, in human AT-MSCs and BM-MSCs grown in different cell culture media.

\begin{tabular}{|c|c|c|c|c|c|}
\hline Staining methods & Day 0 & Day 4 & Day 8 & Day 11 & Day 14 \\
\hline \multicolumn{6}{|c|}{ Anti-DES staining of MSCs } \\
\hline MSCGM & - & + & ++ & ++ & ++ \\
\hline SkGM-2 BulletKit & - & $-/+$ & $-/+$ & - & - \\
\hline 5-Aza & - & $-/+$ & ++ & ++ & ++ \\
\hline \multicolumn{6}{|l|}{ Contidioned medium } \\
\hline $5 \%$ & - & $-/+$ & $+/++$ & $++/+++$ & $++/+++$ \\
\hline $30 \%$ & - & + & $++/+++$ & $++/+++$ & $++/++$ \\
\hline $50 \%$ & - & + & ++ & ++ & ++ \\
\hline \multicolumn{6}{|c|}{ Anti-MYF5 staining of MSCs } \\
\hline MSCGM & - & $+/++$ & + & ++ & ++ \\
\hline SkGM-2 BulletKit & - & + & $-/++$ & ++ & $+++/++++$ \\
\hline $5-\mathrm{Aza}$ & - & + & $-/++$ & +++ & ++ \\
\hline \multicolumn{6}{|l|}{ Contidioned medium } \\
\hline $5 \%$ & - & - & $-/+$ & ++ & $++/+++$ \\
\hline $30 \%$ & - & + & $-/+$ & ++ & $++/+++$ \\
\hline $50 \%$ & - & + & $-/+$ & $+/++$ & ++ \\
\hline
\end{tabular}

The percentage of cells stained by the monoclonal antibodies is symbolised by ' ++++ ' (70-100\%), '+++' (50-70\%), '++' (30-50\%), '+' (1-30\%) and '-' (no staining). DES, desmin; MYF5, myogenic factor 5; MSCs, mesenchymal stem cells; AT-MSCs, MSCs derived from fat/adipose tissue; BM-MSCs, MSCs derived from bone marrow; 5-Aza, 5-azacytidine.

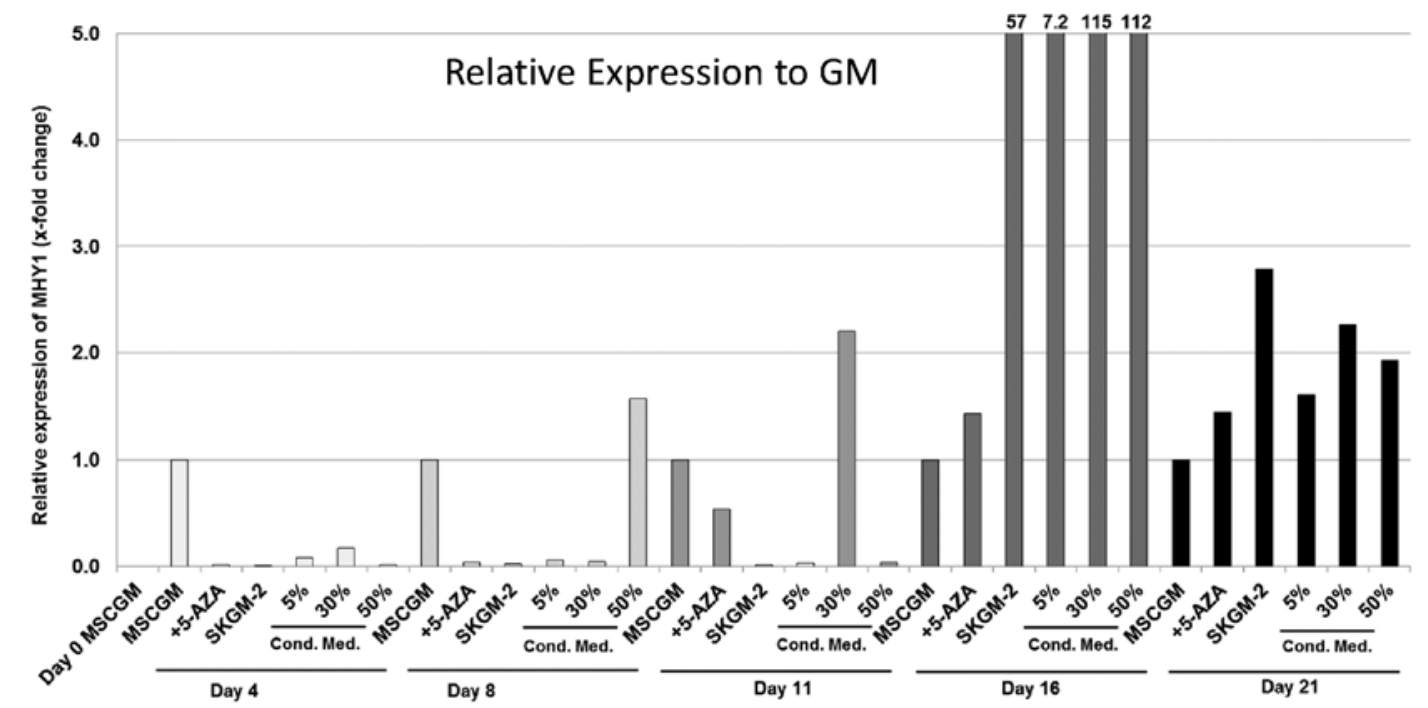

Figure 6. Expression analyses (qRT-PCR) of the myosin, heavy chain 1, skeletal muscle, adult (MYH1) gene in human mesenchymal stem cells (MSCs) derived from fat/adipose tissue (AT-MSCs). Gene expression is presented relative to the expression in MSCGM. The genes were normalised using the normalisation factor (NF) calculated by geNorm software. MSCGM, MSCGM growth medium (GM); 5-Aza, MSCGM + 5-azacytidine (5-Aza); SkGM-2, SkGM-2 BulletKit; $5,30,50 \%$ conditioned cell culture media, supernatant of human satellite cell cultures after three days in cell culture mixed with MSCGM. Cond. Med., conditioned medium.

Following culture in the $30 \%$ conditioned cell culture medium, the amount of DES-positive cells increased to a maximum level on day 8 and remained at this level during the following days (Table II). The percentage of MYF5-positive cells, on the other hand, constantly increased in a time-dependent manner in cell culture. The human AT-MSCs nourished with $50 \%$ conditioned cell culture medium showed lower percentages of DES- and MYF5-positive cells, compared with those cultured with 5 and $30 \%$ conditioned cell culture media, and these levels did not increase (Table II). Analyses of the immunocytochemical staining of the human AT-MSCs cultured in MSCGM, MSCGM + 5-Aza or SkGM-2 BulletKit 

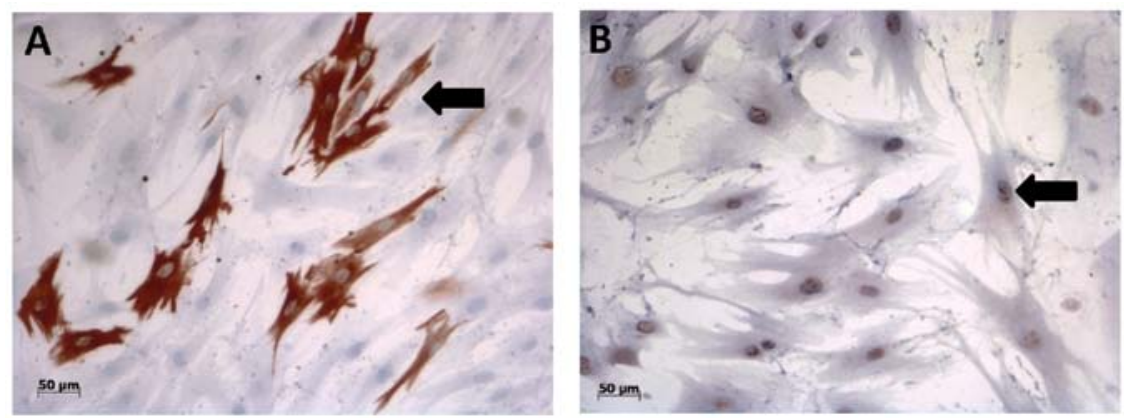

Figure 7. (A) Desmin (DES) and (B) myogenic factor 5 (MYF5) immunoreactivity in human AT-MSCs incubated with 30\% conditioned cell culture medium on day 8. (A) Several DES-immunoreactive human AT-MSCs are shown with immunoreactivity localised in the cytoplasm (arrows indicate DES-positive cells), whereas (B) MYF5 immunoreactivity is localised in the nuclei (arrows indicate MYF5-positive nuclei).

media showed immunoreactive cells, beginning on day 4 of culture. The percentage of immunoreactive AT-MSCs was similar during culture in the three different media. However, the percentage of immunoreactive AT-MSCs was lower than that observed in the cells cultured in 5 and $30 \%$ conditioned cell culture media (Table II).

During the cultivation of BM-MSCs in the different cell culture media, only MYF5 was sporadically detected.

\section{Discussion}

Human MSCs represent a promising alternative stem cell source for skeletal muscle tissue engineering applications, as these cells do not lose their differentiation capacity after cell expansion (5). The myogenic differentiation potential of MSCs has been demonstrated by several research groups; however, it remains unclear whether all cells or only a subpopulation of MSCs can differentiate into skeletal muscle tissue $(21,22)$. MSCs can be isolated from a variety of tissues, which differ in phenotype, differentiation potential and proliferation capacity (10). Several research groups have demonstrated that BM-MSCs, regardless of whether they are injected intravenously or directly into muscle, participate in the regeneration of damaged skeletal muscle in vivo $(23,24)$. However, it should be noted that the detected cell number in the damaged muscle was extremely low (25). These results suggest that a fraction of MSCs are involved in muscle regeneration in vivo and thus, appear promising for in vitro generation of functional skeletal muscle (25). Otherwise, characterised by their high number of pluripotent stem cells, AT-MSCs represent an alternative source of stem cells (26). In addition, AT-MSCs have been detected in skeletal muscle tissue after transplantation into damaged muscle tissue (27), supporting the hypthesis that AT-MSCs contribute to muscle regeneration. The determination of which particular cell line MSCs differentiate into is controlled by multiple factors and extracellular signals, which can be influenced by culture conditions and growth factor compositions. Growth factors and chemical signals that can induce myogenic differentiation in MSCs are bFGF, PDGF, the amino acid, glutamine, and 5-Aza $(5,10,28)$. The underlying mechanisms and pathways have not yet been fully deciphered and are still the subject of investigations. MSCs isolated from different donor sites show similar characteristics in terms of phenotype; however, the ability to differentiate in response to growth factor stimulation differs between MSCs $(8,10)$. De la Garza-Rodea et al demonstrated that myogenic differentiation varied between human MSCs depending on their origin (10). Therefore, in this study, we investigated the myogenic differentiation potential of AT-MSCs and BM-MSCs cultured in different cell culture media with different growth factor compositions in order to detect possible differences in their response to the different cell culture media.

The induction of myogenic differentiation can be initiated using different methods. One method consists of the stimulation of MSCs with 5-Aza, a DNA methyltransferase inhibitor, which leads to the expression of the muscle-specific protein, DES, via the stochastic hypomethylation of random DNA residues (29). However, the myogenic effect of 5-Aza on MSCs remains in question due to findings of other studies (30) and it appears that the effect of 5-Aza depends on cell origin and species (10). The stimulation of myogenic differentiation was achieved in MSCs derived from bone marrow in rats (29), human menstrual blood (31) and the synovial membrane (32). It should be noted that the mode of action and the effect of 5-Aza has not yet been fully elucidated.

Another method of initiation of the myogenic differentiation is the exposure of MSCs to a myogenic environment (33). Due to paracrine secreted cytokines and/or the extracellular matrix, differentiation can be induced in MSCs, although the underlying mechanisms are not yet fully understood. Myogenic differentiation can also be initiated by co-cultivation with myoblasts and the utilisation of conditioned cell culture media in vitro (5). For this reason, we evaluated the effect of the conditioned medium obtained by cultures of human satellite cells grown for three days in MSCGM on the expression of myogenic differentiation markers in human AT-MSCs and BM-MSCs. Furthermore, we evaluated the commercially available MSC culture medium, SkGM-2 BulletKit, which is able to induce myogenic differentiation, which contains the myogenic additives, dexamethasone, FGF and L-glutamine. The exact composition of the medium is known to the manufacturer. MSCGM is a basal growth medium which contains MSCGS and L-glutamine.

To determine the effects of growth factors and media supplements on cell proliferation, an alamarBlue proliferation assay was performed on the human AT-MSC and BM-MSC cultures. In the AT-MSCs, the highest proliferation rates were obtained using the SkGM-2 BulletKit (Fig. 1). The proliferation rate was 3 -fold higher compared with the other cell culture media examined. One reason for this effect may be the higher 
concentration of the epidermal growth factor (rhEGF) in the culture medium, which is known to exert a proliferative effect on cells of mesodermal and ectodermal origin (34). This effect was also detected in the human BM-MSCs, where SkGM-2 BulletKit-treated cells had the highest proliferation rates from day 11 (Fig. 2). Higher rates of cell proliferation were detected in the human AT-MSCs (maximum, 138.2 $\pm 1.66 \mathrm{FU}$ on day 16) compared with the BM-MSCs (maximum, $57.88 \pm 1.26 \mathrm{FU}$ on day 16) following culture in SkGM-2 BulletKit medium. These results support the data of De la Garza-Rodea et al, who demonstrated that AT-MSCs have higher proliferation rates than BM-MSCs (10). This result appears to be consistent in other species, since Nakanishi et al also demonstrated a higher proliferation capacity of AT-MSCs compared with BM-MSCs in rats (35). It should be mentioned that other growth factor compositions were utilised in our experiments. The addition of the DNA methyltransferase inhibitor, 5-Aza, to MSCGM led only to a marginal increase in the proliferation rate of AT-MSCs in comparison to the cells that were cultured in MSCGM. This effect is surprising, since 5-Aza has a cytostatic effect by the demethylation of DNA and RNA. We hypothesised that the cytostatic effect occurs at higher concentrations in human MSCs than the $10 \mu \mathrm{mol} / \mathrm{l}$ that was used, since no apoptotic cells were detected at any time in our preliminary investigations. This result could also not be detected in the BM-MSC. In our study, 5-Aza led to a slight decrease in the proliferation rate. Cytotoxic effects, i.e., cell death, were not detected at any time point. This finding supports the data of Yang et al who demonstrated higher proliferation rates of AT-MSCs compared with BM-MSCs following stimulation with 5-Aza (36) and supports the higher proliferation capacity of human AT-MSCs compared with BM-MSCs.

Beier et al demonstrated the possible myogenic differentiation of MSCs by co-cultivation with myoblasts in rats, verifying the possible paracrine influences on myogenesis (5). Therefore, in this study, we evaluated the effects of conditioned cell culture media, which induced a steady increase in the proliferation rate of AT-MSCs; the concentration (5,30 and 50\%) of the conditioned medium had no effect on the cell proliferation rate. Thus, it can be deduced that the proliferative effects of the growth factors in the conditioned media cannot be enhanced by increasing the concentration. Similar results were obtained in human BM-MSCs, which also showed no significant difference in proliferation with the different conditioned cell culture media, with the exception of the rate on day 8 . These data present the first results on the proliferation capacity of human AT-MSCs and BM-MSCs, investigating the paracrine influences of human myoblasts by different concentrations of conditioned cell culture media.

Using qRT-PCR, we detected the expression of the myogenic marker genes, MYH8, MYH1 and ACTA1, in human AT-MSCs. MYH8 and MYH1 expression was heterogeneous in all the analysed cell culture media with the highest expression values observed in the cells nourished with conditioned media. These results can be explained by the fact that the AT-MSCs represent a heterogeneous group of cells, and not all subpopulations can be driven into the myogenic cell line by medium stimulation (26). Moreover, we detected ACTA1 expression in human AT-MSCs following incubation with each of the six cell culture media examined. The highest expression was measured following culture in $30 \%$ conditioned medium on day 8 . An expression tendency could not be deduced from the expression levels. These results are in accordance with the findings of De la Garza-Rodea et al who also detected a large variety of gene expression of myogenic markers in AT-MSCs (10), supporting the fact that the level of myogenic differentiation is low.

Immunocytochemical staining revealed that the musclespecific intermediate filament, DES, had the highest expression in human AT-MSCs cultured in conditioned media. The highest expression rate was measured following culture in $30 \%$ conditioned medium. Interestingly, DES could only be detected by ICC at very low concentrations in the cells nourished with the commercially available cell culture medium, SkGM-2 BulletKit, which indicates the low degree of myogenic differentiation. This finding underlines the antagonism between proliferation and myogenic differentiation, even in MSCs. Thus, the high proliferation rates seem to be responsible for the lower myogenic differentiation of MSCs.

Moreover, in human BM-MSCs, none of the myogenic differentiation markers were detected, underscoring the low degree of myogenic differentiation of the cells. Immunocytochemical staining of human BM-MSCs revealed no expression of myogenic markers on the protein level and support the gene expression results. These results are in accordance with data of others who also did not detect myogenic differentiation in human adult BM-MSCs following stimulation with 5-Aza (30). The pro-myogenic effect of 5-Aza was achieved in MSCs of rodents and rabbits $(29,37)$ and in human synovial-membrane derived MSCs (32), which provides evidence that MSCs from other species and cell origins respond differently to chemical stimuli, leading to differences in the myogenic differentiation capacity. Chan et al also demonstrated that only in human fetal MSCs, but not in adult BM-MSCs, myogenic differentiation was initiated by cultivation with conditioned cell culture media (30). These data are in accordance with our findings, concerning the absence of myogenic differentiation in BM-MSCs following stimulation with different concentrations of conditioned cell culture media from human satellite cell cultures. According to the data of others, BM-MSCs have been shown to differentiate into functional skeletal muscle only by fusing with pre-determined muscle cells; these data are supported by our results, since in our experiments, no co-cultivation with satellite cells was performed (38). Moreover, it has been shown that adult BM-MSCs cannot form myotubes following implantation into blastocysts, which indicates that these cells are not capable of tissue formation without reprogramming (38).

The induction of myogenic differentiation in human AT-MSCs succeeds only to a certain degree by stimulation with culture media. This conclusion can be derived from our results of gene expression analyses and immunocytochemical investigations, which revealed that the myogenic markers, DES, MYF5, MYH1/8 and ACTA1, were detected in human AT-MSC cultures. The strongest expression of myogenic markers was found in the cells nourished with $30 \%$ conditioned medium. However, it should be noted that the expression of each marker examined could not be detected and no formation of myofibrils was observed.

Our results concerning human AT-MSCs may be explained by the findings of others, who observed that only a small 
subpopulation of MSCs can spontaneously differentiate into skeletal muscle (26), which explains why such heterogeneous results were detected in terms of differentiation marker analysis. According to data of Di Rocco et al, the myogenic phenotype of AT-MSCs can be increased by co-culture with satellite cells (26), providing evidence that the secretion of paracrine growth factors and signalling by muscle precursor cells is a prerequisite for the myogenic differentiation of MSCs. This conclusion is supported by our observation that the highest percentage of cells positive for myogenic markers was found in human AT-MSCs nourished with conditioned cell culture media. Chan et al also observed myogenic differentiation in human fetal MSCs cultured with conditioned cell media in a small population of cells (1-2\%) (30). Moreover, Di Rocco et al also observed that no formation of myofibrils was detected if no cell-cell contacts between muscle cells and MSCs are present (26). Thus, functional skeletal muscle in the sense of multinucleated myofibrils can only be generated by AT-MSCs by fusion with already differentiated myofibrils (26).

In conclusion, it can be stated that SkGM-2 BulletKit led to the highest proliferation rates in AT-MSCs and BM-MSCs and the different concentration of the conditioned cell culture medium had no significant effect on the proliferation of MSCs, regardless of their origin. Furthermore, the evaluation of the effects of six different cell culture media on the myogenic differentiation of human AT-MSCs and BM-MSCs led to the following findings: the myogenic differentiation of human BM-MSCs failed, despite stimulation with different cell culture media and therefore, no myogenic markers (gene expression/protein expression) could be detected. In the human AT-MSCs, myogenic differentiation was initiated to a lower extent following culture in conditioned cell culture media, where the highest expression of the analysed myogenic markers was detected following culture in $30 \%$ conditioned cell culture medium.

\section{Acknowledgements}

The authors would like to thank Michael Collins for assisting with the manuscript, as well as Petra Prohaska and Ulrike Traut for their excellent technical assistance. This study contains part of the doctoral thesis of Stephanie Juritz.

\section{References}

1. Belema Bedada F, Technau A, Ebelt H, Schulze M and Braun T: Activation of myogenic differentiation pathways in adult bone marrow-derived stem cells. Mol Cell Biol 25: 9509-9519, 2005.

2. Zuk PA, Zhu M, Mizuno H, et al: Multilineage cells from human adipose tissue: implications for cell-based therapies. Tissue Eng 7: 211-228, 2001.

3. Bieback K, Kern S, Kluter H and Eichler H: Critical parameters for the isolation of mesenchymal stem cells from umbilical cord blood. Stem Cells 22: 625-634, 2004.

4. Igura K, Zhang X, Takahashi K, Mitsuru A, Yamaguchi S and Takashi TA: Isolation and characterization of mesenchymal progenitor cells from chorionic villi of human placenta. Cytotherapy 6: 543-553, 2004.

5. Beier JP, Bitto FF, Lange C, et al: Myogenic differentiation of mesenchymal stem cells co-cultured with primary myoblasts. Cell Biol Int 35: 397-406, 2011.

6. Garcia-Castro J, Trigueros C, Madrenas J, Perez-Simon JA, Rodriguez R and Menendez P: Mesenchymal stem cells and their use as cell replacement therapy and disease modelling tool. J Cell Mol Med 12: 2552-2565, 2008
7. Pittenger MF and Martin BJ: Mesenchymal stem cells and their potential as cardiac therapeutics. Circ Res 95: 9-20, 2004.

8. Meligy FY, Shigemura K, Behnsawy HM, Fujisawa M, Kawabata $\mathrm{M}$ and Shirakawa T: The efficiency of in vitro isolation and myogenic differentiation of MSCs derived from adipose connective tissue, bone marrow, and skeletal muscle tissue. In Vitro Cell Dev Biol Anim 48: 203-215, 2012.

9. Vidane AS, Zomer HD, Oliveira BM, et al: Reproductive stem cell differentiation: extracellular matrix, tissue microenvironment, and growth factors direct the mesenchymal stem cell lineage commitment. Reprod Sci 20: 1137-1143, 2013.

10. de la Garza-Rodea AS, van der Velde-van Dijke I, Boersma H, et al: Myogenic properties of human mesenchymal stem cells derived from three different sources. Cell Transplant 21: 153-173, 2012.

11. Sordella R, Jiang W, Chen GC, Curto M and Settleman J: Modulation of Rho GTPase signaling regulates a switch between adipogenesis and myogenesis. Cell 113: 147-158, 2003.

12. Tapscott SJ and Weintraub H: MyoD and the regulation of myogenesis by helix-loop-helix proteins. J Clin Invest 87: 1133-1138, 1991.

13. Christ B and Brand-Saberi B: Limb muscle development. Int $\mathbf{J}$ Dev Biol 46: 905-914, 2002.

14. Stern-Straeter J,Bonaterra GA,Kassner SS, et al: Characterization of human myoblast differentiation for tissue-engineering purposes by quantitative gene expression analysis. J Tissue Eng Regen Med 5: e197-e206, 2011.

15. Wright C, Haddad F, Qin AX and Baldwin KM: Analysis of myosin heavy chain mRNA expression by RT-PCR. J Appl Physiol (1985) 83: 1389-1396, 1997.

16. Wehrle U, Dusterhoft S and Pette D: Effects of chronic electrical stimulation on myosin heavy chain expression in satellite cell cultures derived from rat muscles of different fiber-type composition. Differentiation 58: 37-46, 1994.

17. Goessler UR, Bugert $\mathrm{P}$, Bieback K, et al: Integrin expression in stem cells from bone marrow and adipose tissue during chondrogenic differentiation. Int J Mol Med 21: 271-279, 2008.

18. Stern-Straeter J, Bonaterra GA, Kassner SS, et al: Impact of static magnetic fields on human myoblast cell cultures. Int J Mol Med 28: 907-917, 2011.

19. Vandesompele J, De Preter K, Pattyn F, et al: Accurate normalization of real-time quantitative RT-PCR data by geometric averaging of multiple internal control genes. Genome Biol 3: RESEARCH0034, 2002.

20. Stern-Straeter J, Bonaterra GA, Hormann K, Kinscherf R and Goessler UR: Identification of valid reference genes during the differentiation of human myoblasts. BMC Mol Biol 10: 66, 2009.

21. Dezawa M, Ishikawa H, Itokazu Y, et al: Bone marrow stromal cells generate muscle cells and repair muscle degeneration. Science 309: 314-317, 2005.

22. Corti S, Strazzer S, Del Bo R, et al: A subpopulation of murine bone marrow cells fully differentiates along the myogenic pathway and participates in muscle repair in the mdx dystrophic mouse. Exp Cell Res 277: 74-85, 2002.

23. LaBarge MA and Blau HM: Biological progression from adult bone marrow to mononucleate muscle stem cell to multinucleate muscle fiber in response to injury. Cell 111: 589-601, 2002.

24. Ferrari G and Mavilio F: Myogenic stem cells from the bone marrow: a therapeutic alternative for muscular dystrophy? Neuromuscul Disord 12 (Suppl 1): S7-S10, 2002.

25. Ferrari G, Cusella-De Angelis G, Coletta M, et al: Muscle regeneration by bone marrow-derived myogenic progenitors. Science 279: 1528-1530, 1998.

26. Di Rocco G, Iachininoto MG, Tritarelli A, et al: Myogenic potential of adipose-tissue-derived cells. J Cell Sci 119: 2945-2952, 2006.

27. Miranville A, Heeschen C, Sengenes C, Curat CA, Busse R and Bouloumie A: Improvement of postnatal neovascularization by human adipose tissue-derived stem cells. Circulation 110: 349-355, 2004.

28. Gang EJ, Jeong JA, Hong SH, et al: Skeletal myogenic differentiation of mesenchymal stem cells isolated from human umbilical cord blood. Stem Cells 22: 617-624, 2004.

29. Wakitani S, Saito T and Caplan AI: Myogenic cells derived from rat bone marrow mesenchymal stem cells exposed to 5-azacytidine. Muscle Nerve 18: 1417-1426, 1995.

30. Chan J, O'Donoghue K, Gavina M, et al: Galectin-1 induces skeletal muscle differentiation in human fetal mesenchymal stem cells and increases muscle regeneration. Stem Cells 24: 1879-1891, 2006 
31. Cui CH, Uyama T, Miyado K, et al: Menstrual blood-derived cells confer human dystrophin expression in the murine model of Duchenne muscular dystrophy via cell fusion and myogenic transdifferentiation. Mol Biol Cell 18: 1586-1594, 2007.

32. De Bari C, Dell'Accio F, Tylzanowski P and Luyten FP: Multipotent mesenchymal stem cells from adult human synovial membrane. Arthritis Rheum 44: 1928-1942, 2001.

33. Lee JH, Kosinski PA and Kemp DM: Contribution of human bone marrow stem cells to individual skeletal myotubes followed by myogenic gene activation. Exp Cell Res 307: 174-182, 2005.

34. Tamama K, Kawasaki $\mathrm{H}$ and Wells A: Epidermal growth factor (EGF) treatment on multipotential stromal cells (MSCs). Possible enhancement of therapeutic potential of MSC. J Biomed Biotechnol 2010: 795385, 2010.
35. Nakanishi C, Nagaya N, Ohnishi S, et al: Gene and protein expression analysis of mesenchymal stem cells derived from rat adipose tissue and bone marrow. Circ J 75: 2260-2268, 2011.

36. Yang J, Song T, Wu P, et al: Differentiation potential of human mesenchymal stem cells derived from adipose tissue and bone marrow to sinus node-like cells. Mol Med Rep 5: 108-113, 2012.

37. Rangappa S, Fen C, Lee EH, Bongso A and Sim EK: Transformation of adult mesenchymal stem cells isolated from the fatty tissue into cardiomyocytes. Ann Thorac Surg 75: 775-779, 2003

38. Schulze M, Belema-Bedada F, Technau A and Braun T: Mesenchymal stem cells are recruited to striated muscle by NFAT/ IL-4-mediated cell fusion. Genes Dev 19: 1787-1798, 2005. 